\title{
CO concentration and temperature measurements in a shock tube for Martian mixtures by coupling OES and TDLAS
}

\author{
X. Lin $\cdot$ X. L. Yu $\cdot$ F. Li $\cdot$ S. H. Zhang \\ J. G. Xin $\cdot$ X. Y. Chang
}

Received: 20 July 2012/Revised: 8 November 2012/Published online: 23 November 2012

(C) Springer-Verlag Berlin Heidelberg 2012

\begin{abstract}
CO}$ concentration and gas temperature distribution are diagnosed behind a strong shock wave simulating the Martian atmosphere entry processes by coupling optical emission spectroscopy (OES) and tunable diode laser absorption spectroscopy (TDLAS). The strong shock wave $(6.31 \pm 0.11 \mathrm{~km} / \mathrm{s})$ is established in a shock tube driven by combustion of hydrogen and oxygen. Temperature of the shock-heated gas is inferred through a precise analysis of the high temporal and spatial resolution experimental spectral of $\mathrm{CN}$ violet system $\left(B^{2} \Sigma^{+} \rightarrow X^{2} \Sigma^{+}\right.$, $\Delta v=0$ sequence) using OES. A CO absorption line near 2,335.778 $\mathrm{nm}$ is utilized for detecting the $\mathrm{CO}$ concentration using scanned-wavelength direct absorption mode with $50 \mathrm{kHz}$ repetition rate. Combined with temperature results from OES, $\mathrm{CO}$ concentration in the thermal equilibrium region is derived. The current experimental results are complementary for determining an accurate rate coefficient of $\mathrm{CO}_{2}$ dissociation and validation relevant chemical kinetics models in Mars atmosphere entry processes.
\end{abstract}

\section{Introduction}

The escape velocity for the plant Mars is $5.0 \mathrm{~km} / \mathrm{s}$. Minimum-energy flight trajectories from Earth to Mars result in

X. Lin · J. G. Xin

School of Optoelectronics, Beijing Institute

of Technology, Beijing 100081, China

X. Lin $\cdot$ X. L. Yu $(\bowtie) \cdot$ F. Li $\cdot$ S. H. Zhang $\cdot$ X. Y. Chang

State Key Laboratory of High Temperature Gas

Dynamics, Institute of Mechanics, Chinese Academy

of Sciences, Beijing 100190, China

e-mail: xlyu@imech.ac.cn a Martian entry velocity of $5.8 \mathrm{~km} / \mathrm{s}$ or much higher [1], which will generate a bow shock in front of the heat shield of a space probe. In the shock layer, physical and chemical phenomena produce a non-equilibrium situation giving birth to a strong radiative emission from the excited species $[2,3]$. It is well known that in those conditions main radiative systems include $\mathrm{C}_{2}$ Swan bands, the $\mathrm{CO}$ fourth positive system, the $\mathrm{CN}$ red system, as well as the $\mathrm{CN}$ violet system. The radicals contribute significantly to radiative heating rates during entries to Mars, which has $\mathrm{CO}_{2} / \mathrm{N}_{2}$ atmosphere. Therefore, future exploration missions towards the Mars need further understanding of physical and chemical processes occurring during the probe entry in the upper atmosphere.

So far, the physical and chemical processes behind a reentry shock wave, especially about non-equilibrium radiation and chemical reaction occurring in the shock layer, can only be reproduced in some ground-based simulation facilities such as shock tube or shock tunnel [4]. Experimental investigations of the processes in shockheated simulated Martian mixtures using different kinds of shock tubes recently were reported by a number of institutions, including NASA Ames Research Center in California [5, 6], Université de Provence in Marseilles, France [7-9], and University of Queensland in Australia [10, 11]. NASA Ames used an electric arc-driven shock tube facility known as EAST, whereas Université de Provence and University of Queensland's tests were on free-piston-driven shock tubes based on the Stalker principle [12], known as TCM2 and $\mathrm{X} 2$, respectively. Until now, the radiation measurements have been widely investigated through optical emission spectroscopy (OES), and several thermodynamic models have been established to predict nonequilibrium radiation and kinetic phenomena occurring in the shock layer. All these results are useful to describe 
Mars atmosphere entry process and to validate relevant chemical models.

However, the physical and chemical models still need to be validated. It is well known that Mars atmosphere is mainly composed of $\mathrm{CO}_{2}(97 \%)$. The processes occurring in the shock layer, especially about temperature distribution and carbon dioxide dissociation are not completely resolved. That one of the most important reactions is the dissociation of $\mathrm{CO}_{2}$ encountered for space probe's reentry is shown as following,

$\mathrm{CO}_{2}+M \rightarrow \mathrm{CO}+\mathrm{O}+M$

$\mathrm{CO}+M \rightarrow \mathrm{C}+\mathrm{O}+M$

where the rate coefficient of rate-limiting reaction (1) for dissociation processes is much more significant [1]. Temperature is one of the most important thermodynamic quantities in shock-induced chemistry because it is a key parameter in chemical reactions. Therefore, accurate quantitative measurement of carbon monoxide concentration and temperature along the strong shock wave will help to understand carbon dioxide dissociation and validate the relevant chemical kinetic models [13, 14]. Consequently, development of accurately quantitative diagnostic techniques for shock tube experiments is necessary. As we know, OES is widely used for temperature estimation of self-luminous high-temperature gas behind a strong shock wave because it is non-intrusive, high sensitivity, and just simple instruments constitute [5-11]. Tunable diode laser absorption spectroscopy (TDLAS) is another effective method for measuring gas temperature and concentration in many fields due to its advantage of non-intrusive, high sensitivity, gas-specific and quick response $[15,16]$. However, according to our knowledge, there is no relevant literature of TDLAS monitoring CO behind normal shock waves for Mars atmosphere entry testing.

In this paper, $\mathrm{CO}$ concentration and gas temperature distribution behind a strong shock wave were simultaneously measured by an optical diagnostic system based on OES and TDLAS. Through OES diagnostics, high temporal and spatial resolution experimental spectra of $\mathrm{CN}$ violet system $\left(B^{2} \Sigma^{+} \rightarrow X^{2} \Sigma^{+}, \Delta v=0\right.$ sequence) were obtained with ICCD gating time as short as $50 \mathrm{~ns}$. The temperature distribution along shock profile can be directly obtained through a precise analysis of high-resolution experimental spectra. The processing method was described in detail in the literature [17]. The TDLAS system was operated in scanned-wavelength direct absorption mode to diagnose one $\mathrm{CO}$ absorption line near 2,335.778 $\mathrm{nm}$. Absorption measurement experiments were first conducted in a static cavity to calibrate the absorption line near $2,335.778 \mathrm{~nm}$ by the well-known position of the strong $\mathrm{CO}$ absorption line near $2,335.544 \mathrm{~nm}$ at room temperature. Then, experiments were carried out in a hydrogen-oxygen combustion driven shock tube. According to the result from OES and TDLAS, CO concentration in the thermal equilibrium region, can be determined. To our knowledge, this is the first quantitative absorption measurement of $\mathrm{CO}$ concentration at relevant conditions for Mars atmospheric entry.

\section{Absorption spectroscopy theory and line selection}

The fundamental theory of direct-absorption spectroscopy has been detailed by numerous researchers [18, 19], and is reproduced here briefly to define units and terms. The fractional absorption of the laser intensity $\Delta I / I_{0}$ is related to the absorption coefficient $k_{\mathrm{v}}$ by the Beer-Lambert law expressed as

$\left(\frac{\Delta I}{I_{0}}\right)_{\mathrm{LAS}}=\frac{I_{0}-I_{\mathrm{t}}}{I_{0}}=\exp \left(-k_{\mathrm{v}} L\right)$

where $I_{0}$ and $I_{\mathrm{t}}$ are the incident and transmitted laser intensities; $L[\mathrm{~cm}]$ is the absorbing pass length; $k_{\mathrm{v}}\left[\mathrm{cm}^{-1}\right]$ is the spectral absorption coefficient. For an isolated absorption transition, the spectral absorption coefficient $k_{\mathrm{v}}$ can be expressed as

$k_{\mathrm{v}}=P_{\mathrm{CO}} \cdot S(T) \cdot \phi(v)$

where $P_{\mathrm{CO}}[\mathrm{atm}]$ is the partial pressure of absorbing species, $S(T)\left[\mathrm{cm}^{-2} \mathrm{~atm}^{-1}\right]$ and $\phi(v)[\mathrm{cm}]$ are the line strength and line-shape function, respectively. The product $k_{\mathrm{v}} L$ is known as the spectral absorbance $\alpha_{\mathrm{v}}$.

$\alpha_{\mathrm{v}} \equiv-\ln \left(\frac{\Delta I}{I_{0}}\right)=k_{\mathrm{v}} L=P_{\mathrm{CO}} S(T) \phi(v) L$

The line-shape function $\phi(v)$ is usually approximated using a Voigt profile which is a convolution of the Gaussian and Lorentz profiles that represent two significant broadening mechanisms. The line-shape function $\phi(v)$ is normalized to unity (defined as $\int \phi(v) d v \equiv 1$ ), so that if the temperature $T[\mathrm{~K}]$ is acquired and the wavelength scans strategy is performed, by integrating absorbance over the whole wavelength range, absorbing species partial pressure $P_{\mathrm{CO}}$ can be found.

$P_{\mathrm{CO}}=\frac{\int \alpha_{\mathrm{v}} d v}{S(T) L}$

Based on the ideal gas equation, we can obtain the concentration of absorbers $n_{i}$, which is related to $T$ as 
$n_{i}=\frac{6.02 \times 10^{23} \cdot P_{\mathrm{CO}}}{R \cdot T} \mathrm{~m}^{-3}=\frac{6.02 \times 10^{17} \cdot \int \alpha_{\mathrm{v}} d v}{R \cdot T \cdot S(T) \cdot L} \mathrm{~cm}^{-3}$

Here, $R[\mathrm{~J} /(\mathrm{mol} \mathrm{K})]$ is the gas constant. The line strength at any temperature, $S(T)$, can be calculated from the known line strength at some reference temperature, $S\left(T_{0}\right)$, using

$$
\begin{aligned}
S(T)= & S\left(T_{0}\right) \frac{Q\left(T_{0}\right)}{Q(T)}\left(\frac{T_{0}}{T}\right) \exp \left[-\left(\frac{h c}{k}\right)\left(E_{1}^{\prime \prime}-E_{2}^{\prime \prime}\right)\left(\frac{1}{T}-\frac{1}{T_{0}}\right)\right] \\
& \frac{\left[1-\exp \left(-\frac{h c v_{0}}{k T}\right)\right]}{\left[1-\exp \left(-\frac{h c v_{0}}{k T_{0}}\right)\right]}
\end{aligned}
$$

where $h[\mathrm{~J} \mathrm{~s}]$ is Planck's constant, $c[\mathrm{~cm} / \mathrm{s}]$ is the speed of light, $k[\mathrm{~J} / \mathrm{K}]$ is Boltzmann's constant, the total partition function of the molecular, $Q(T)$, of $\mathrm{CO}$ can be calculated and have been reported in the literature [20,21], and $E^{\prime \prime}$ $\left[\mathrm{cm}^{-1}\right]$ is the lower state energy of the transition. Here, we use the $296 \mathrm{~K}$ as the reference temperature, while the corresponding line strength $S(296)$ comes from the HITRAN2008 database.

Before absorption line selecting, we should analyze the region of gas flow parameters. In our experiments, the absorption length is fixed at $7.8 \mathrm{~cm}$. Based on thermodynamic calculations, the pressure after a strong shock wave is about $1.1 \mathrm{~atm}$. Temperature in the thermal equilibrium region behind the strong shock wave is inferred $7,400 \pm 300 \mathrm{~K}$ by OES. In such a high temperature environment, it is a challenge to calibrate the absolute line positions. Therefore, absorption spectra based on the HITRAN2008 database near $2.3 \mu \mathrm{m}$ are computed for choosing appropriate $\mathrm{CO}$ transitions, as shown in Fig. 1. The CO absorption line near 2,335.778 nm is selected because it can be conveniently calibrated by the absorption line near $2,335.544 \mathrm{~nm}$, which can be easily verified by a

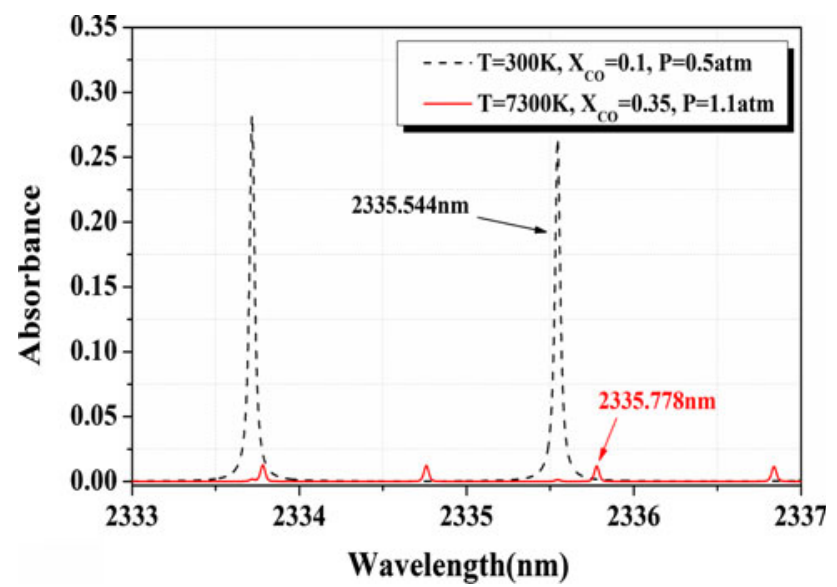

Fig. 1 Spectral simulation of CO absorption near $2.3 \mu \mathrm{m}$ for choosing appropriate $\mathrm{CO}$ transitions wavelength meter and a Fabry-Perot interferometer at room temperature.

\section{Facility and experimental set-up}

\subsection{Static cavity}

Absorption measurements of the $\mathrm{CO}$ absorption line near $2,335.544 \mathrm{~nm}$ in a room temperature cavity are used to calibrate the absorption line near $2,335.778 \mathrm{~nm}$. The arrangement of the experiment is shown in Fig. 2. A distributed-feedback laser (manufactured by Nanoplus $\mathrm{GmbH}$ ), which is controlled by commercial temperature and current controllers (Thorlabs, Model: ITC-502), is lasing the wavelength near $2.3 \mu \mathrm{m}$ for this study. A function generator (Tektronix, Model: AFG-3101) is used to ramp the laser injection current and thus tune the wavelengths of the laser over the desired absorption features. The laser beam is collimated by an achromatic doublet lens. A gold mirror is used to adjust the beam across the quartz windows on both sides of the static cavity. Laser modulation parameters are the same in both the static cavity and the shock tube experiments. Here, a scannedwavelength direct absorption is used. The wavelength is tuned over a range of $0.7 \mathrm{~nm}$ and the repetition frequency is set at $50 \mathrm{kHz}$, while the signals are sensed by two InGaAs detectors (Thorlabs, Model: DET10D) and recorded by a memory oscilloscope (Tektronix Inc., Model: DPO4034) with $100 \mathrm{MS} / \mathrm{s}$ sampling rate. Using two beam splitters, the two beams of reflected light are simultaneously steered to pass through a Fabry-Perot interferometer (Thorlabs, Model: SA200-18B, 1.5 GHz FSR) and a free-space mid-IR wavelength meter (Bristol, Model: 721) for wavelength marking, these measurements also provide absolute line positions which are in excellent agreement with the HITRAN2008 database.

\subsection{Shock tube}

The experimental investigations are carried out in a hydrogen-oxygen combustion shock tube facility. Figure 3 gives an overview of the facility with essential dimensional details and an optical diagnostic system. The internal diameter of the stainless steel shock tube is $78 \mathrm{~mm}$. It is equipped with three ion probes that record the shock front during a run, so that the shock velocity can be measured. Two $10 \mathrm{~mm}$ diameter quartz windows are equipped on both sides of the shock tube to carry out optical measurements. The windows are wedged at $3^{\circ}$ angle to avoid unwanted interference fringes. More detailed description of the facility and its functioning is given in the literature [17]. In our experiments, the mixture consists of $70 \% \mathrm{CO}_{2}$ 
Fig. 2 Schematic diagram of the experimental set-up for calibration the $\mathrm{CO}$ absorption line near 2,335.778 $\mathrm{nm}$ at controlled pressure and room temperature
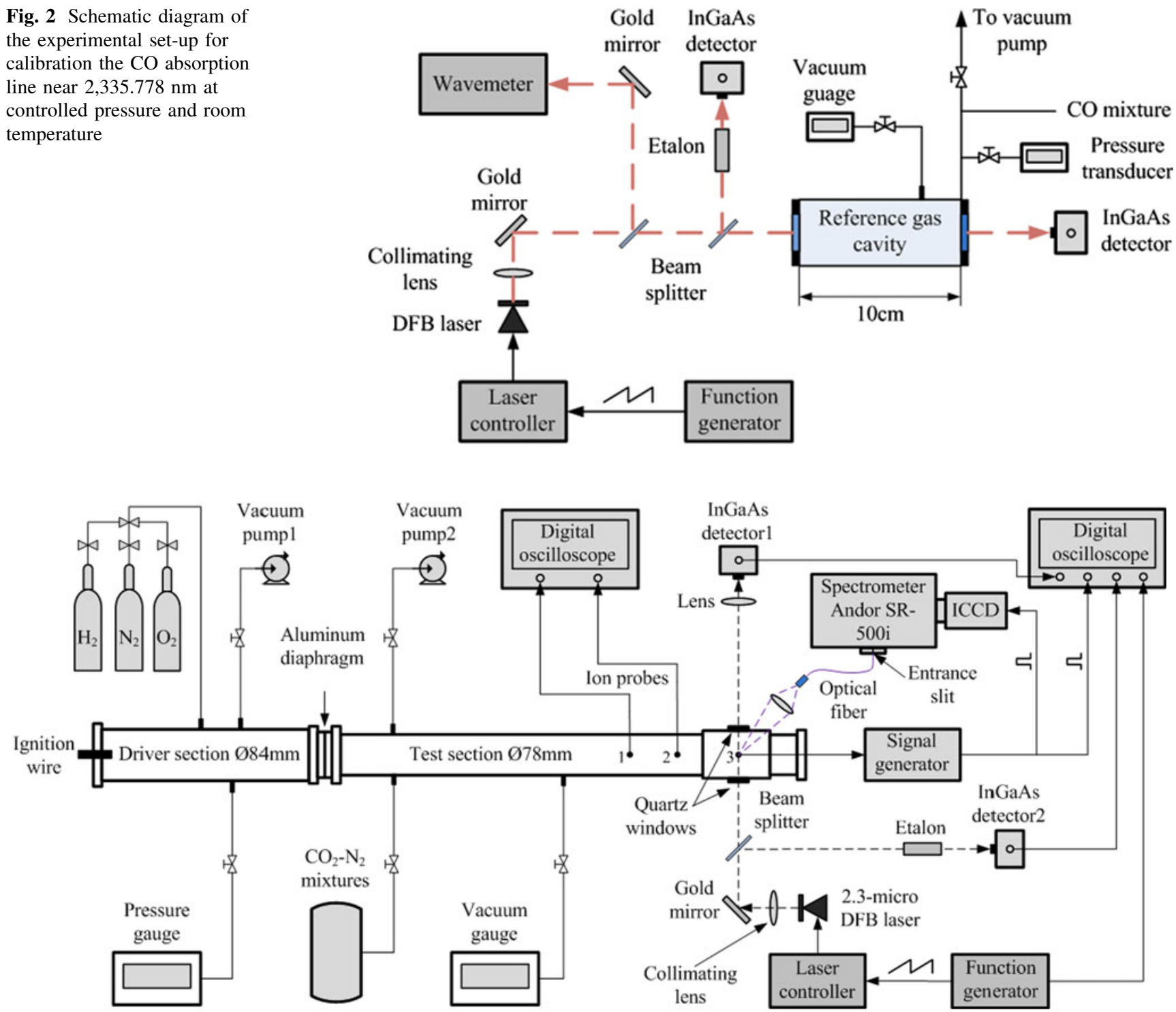

Fig. 3 Schematic diagram of the experimental set-up of the shock tube and the optical instruments arrangement

and $30 \% \mathrm{~N}_{2}$. Despite the difference from the actual composition of the Mars atmosphere, such a ratio can significantly improve the signal-to-noise (SNR) of the $\mathrm{CN}$ radicals emission while the chemical kinetics processes remain the same. The pressure is $200 \mathrm{~Pa}$ and the average shock velocity is equal to $6.31 \mathrm{~km} / \mathrm{s}$ with $1.7 \%$ variation.

The optical diagnostic system consists of two parts: the TDLAS system and the OES system, where the TDLAS system is similar to the one described for the static cavity calibrations except that the transmission light is focused by a lens in the shock tube experiment. The lens could effectively enhance the detection signal even in the presence of various beam-steering and misalignment. The OES system arrangement during these experiments is designed to allow the recording of the $\mathrm{CN}$ violet system radiation emitted from the hot test gas immediately following the shock front. The emission has been collected through an optical fiber on which is focused the light coming through a lens system. The role of the lens system is to ensure an acceptable spatial resolution with $1 \mathrm{~mm}$ in diameter and $1 \mathrm{~mm}$ in depth. Spectral information is recorded using an Andor SR-500i $500 \mathrm{~mm}$ focal length imaging, triple grating spectrometer coupled to an Andor DH-740 $2048 \times$ 512 pixels gated intensified charge couple device (ICCD). A 1,200-grooves/mm grating, together with an entrance slit aperture of $100 \mu \mathrm{m}$ are used in the spectrometer giving a spectral resolution of $0.154 \mathrm{~nm}$ (full-width at half-maximum), which is determined experimentally by comparing atomic lines of the experimental and theoretical spectra of a narrow line width diode laser (center wavelength: $777.19 \mathrm{~nm}$, line width $\Delta \lambda<0.001 \mathrm{~nm}$ ). The TDLAS system and OES system are triggered simultaneously by 
the No. 3 ion probe through a signal generator (Stanford Research System, Model: DG535) when the shock wave nears the location No. 3 ion probe which is installed on the same section as spectrum capture field. Due to the very quick response of the ion probe $(\sim 100 \mathrm{~ns})$ and very short internal time delay of electric circuit in the camera ( $\sim 50 \mathrm{~ns}$ ), the ion triggering system enabled us to accurately capture the flow area of interest. The camera exposure time is set as $50 \mathrm{~ns}$, which means the shock wave only moves about $0.12 \mathrm{~mm}$, much smaller than the spectral capture field. Because the time between two acquisitions limited by the computer storage frequency, cannot be $<0.01 \mathrm{~s}$, it means that only single spectrum can be recorded during one shot. The delay of the gate pulse can be tuned to record different moments so that the spectrum is to be captured in the equilibrium, or in the non-equilibrium region.

The acquired spectra are calibrated in relative intensity in the UV range (below $400 \mathrm{~nm}$ ) with a NIST traceable quartz-tungsten-halogen standard light source (Oriel, Model: 63945). The calibration procedure is carried outside the shock tube because of the large size of the tungsten lamp, and the effect of the observation window, optical fiber, grating and ICCD camera is also taken into account. The signals' output from ion probes, InGaAs detectors and the function generator are simultaneously recorded by a high dynamic memory digital oscilloscope (Tektronix Inc., Model: DPO4034).

\section{Results and discussions}

\subsection{Wavelength calibration for the TDLAS system}

The TDLAS system is first validated in the static cavity at room temperature before being used in shock tube. Laser modulation parameters are the same in the room temperature cavity and the shock tube experiments. Since the typical test times of interest in our shock tube experiments are on the magnitude of $100 \mu \mathrm{s}$, hence fast time response is needed for measurements. The scan rate using a ramp signal $(50 \mathrm{kHz})$ is chosen as a tradeoff between acquiring enough data points per scan with sampling resolution and generating enough scans per test in the $100 \mu$ s run duration. Although the time to cover the absorption feature is about $1.6 \mu \mathrm{s}$, the temporal resolution of absorption measurement is sufficient for the diagnosis in the equilibrium region because whose effective time is at least $12 \mu$ s by the OES.

This absorption measurement, combined with relative measurements using a Fabry-Perot interferometer, provides the absolute position of the absorption line near $2,335.778 \mathrm{~nm}$, as shown in Fig. 4. In addition, the absorption line near $2,335.544 \mathrm{~nm}$ can also be used to

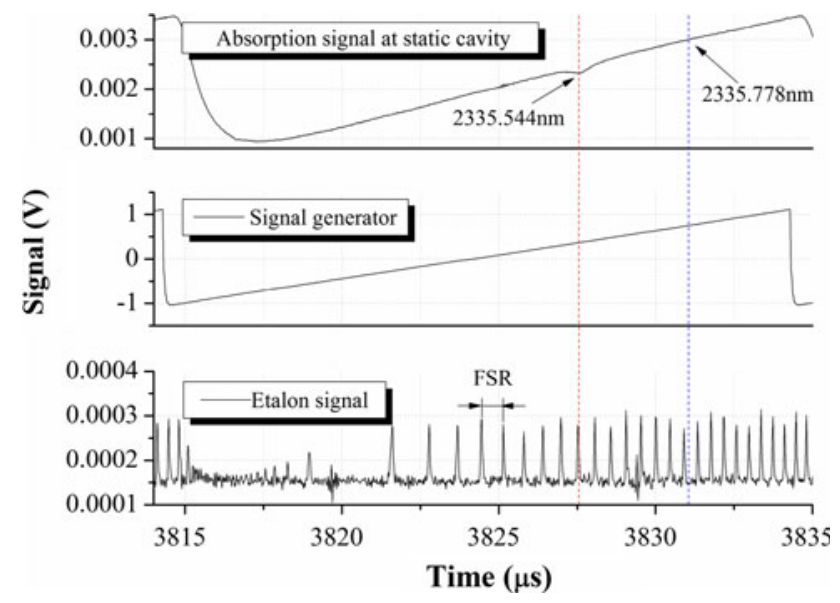

Fig. 4 The CO absorption line near 2,335.778 $\mathrm{nm}$ is calibrated by the known position of the strong $\mathrm{CO}$ absorption line near 2,335.544 nm at a room temperature cavity

check the process of changing the absorption scans in time to wavenumber as the line positions of these transitions are quite accurately known.

\subsection{Rotational and vibrational temperatures determination}

Time-resolved spectra of the $\Delta v=0$ sequence of the $B^{2} \Sigma^{+} \rightarrow X^{2} \Sigma^{+}$electronic transition of $\mathrm{CN}$ have been observed through OES. $\mathrm{CN}$ radicals are among the best molecules for temperature measurements of high-temperature gas because their emission efficiency is very high and they are stable among a wide range of chemical compositions at high temperature. In our studies, $\mathrm{CN}$ is a signature of the chemical reaction between $\mathrm{CO}_{2}$ and $\mathrm{N}_{2}$, thus, it is feasible and important in estimating the temperature using $\mathrm{CN}$ radicals in the high-temperature gas mixture behind a strong shock wave.

First of all, we analyze in detail the spectral structure of $\mathrm{CN}$ violet system $\left(B^{2} \Sigma^{+} \rightarrow X^{2} \Sigma^{+}, \Delta v=0\right.$ sequence $)$ and propose a rotational temperature measurement method based on fitting high-resolution spectrum. The similar method has also been employed by other researcher $[7,8$, 24]. Here, we will briefly introduce rotational temperature and vibrational temperature determination through pattern match scheme by OES. Figure 5 illustrates the structure of the different vibrational band spectra and theoretical synthetic spectra of the $\mathrm{CN}$ violet band system $\left(B^{2} \Sigma^{+} \rightarrow X^{2} \Sigma^{+}\right.$, $\Delta v=0$ sequence) at 7,000 $\mathrm{K}$ in thermal equilibrium. The rectangular panel shows that the range $(387.6-388.6 \mathrm{~nm})$ of the emission spectrum of the $\mathrm{CN}$ violet system is completely controlled by the $(0,0)$ transition, in other words, this distribution pattern of a sequence is controlled only by rotational temperature $T_{\text {rot }}$. This wavelength range corresponds to emission lines of the $P$ branch (rotational 


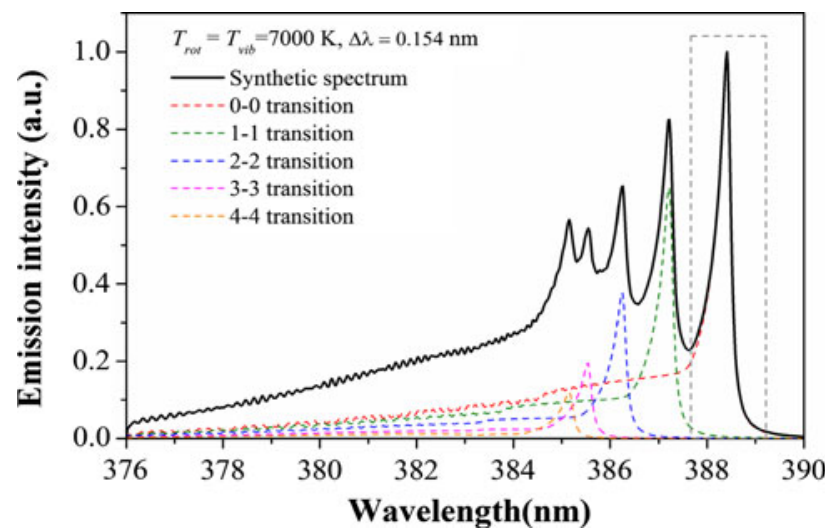

Fig. 5 Structure of theoretical synthetic spectra in the $\mathrm{CN}$ violet system $\left(B^{2} \Sigma^{+} \rightarrow X^{2} \Sigma^{+}, \Delta v=0\right)$

number $J$ range from 0 to 57), and the slight effect of other transitions tends to be neglected, as shown in Fig. 5. According to the measured spectral resolution, the $T_{\text {rot }}$ is determined by comparing this fraction of the wavelength range between the experimental and theoretical synthetic spectra. The uncertainty of the rotational temperature measurement mainly comes from the theoretical spectrum calculation. We discuss in detail as follows.

The emission intensity is described as

$I_{v^{\prime \prime}, J^{\prime \prime}}^{v^{\prime}, J^{\prime}}=N_{v^{\prime}, J^{\prime}} A_{v^{\prime \prime}, J^{\prime \prime}}^{v^{\prime}, J^{\prime}} h v_{v^{\prime \prime}, J^{\prime \prime}}^{v^{\prime}, J^{\prime}}$

Assuming Born-Oppenheimer approximation and Frank-Condon principle, the transition probability is expressed as

$A_{v^{\prime \prime}, J^{\prime \prime}}^{v^{\prime}, J^{\prime}}=\frac{64 \pi\left(v_{v^{\prime \prime}, J^{\prime \prime}}^{v^{\prime}, J^{\prime}}\right)^{3} S_{J^{\prime \prime}}^{J^{\prime \prime}}}{3 h\left(2 J^{\prime}+1\right)} q_{v^{\prime}, v^{\prime \prime}} R_{\mathrm{e}}^{2}$

where $\left(v^{\prime}, J^{\prime}\right)$ and $\left(v^{\prime \prime}, J^{\prime \prime}\right)$ represent the vibrational and rotational quantum numbers in the upper level and lower lever, respectively; $R_{\mathrm{e}}^{2}$ is the average value of the electronic transition moment and can be considered to be constant; $q_{v^{\prime}, v^{\prime \prime}}$ is the Franck-Condon factor, $S_{J^{\prime \prime}}^{J^{\prime}}$ is the Hönl-London factor, and $v_{v^{\prime \prime}}^{v^{\prime}, J^{\prime}}$ is the transition frequency. Assuming that the rotational state of $\mathrm{CN}$ is in thermal equilibrium, fixing the value of $v^{\prime}$ and $v^{\prime \prime}$, and substituting Eq. (10) to Eq. (9), the rotational term of the emission intensity is described by

$I_{v^{\prime \prime}, J^{\prime \prime}}^{v^{\prime}, J^{\prime}} \propto\left(v_{v^{\prime \prime}, J^{\prime \prime}}^{v^{\prime}, J^{\prime}}\right)^{4} S_{J^{\prime \prime}}^{J^{\prime}} \exp \left(-\frac{F\left(J^{\prime}\right)}{k T_{\text {rot }}}\right)$

The rotational energy and the transition frequency are given by

$F\left(v^{\prime}, J^{\prime}\right)=B_{v^{\prime}} J^{\prime}\left(J^{\prime}+1\right)-D_{v^{\prime}} J^{\prime 2}\left(J^{\prime}+1\right)^{2}$

$B_{v^{\prime}}=B_{\mathrm{e}}-\alpha_{\mathrm{e}}\left(v^{\prime}+\frac{1}{2}\right)$
$D_{v^{\prime}}=D_{\mathrm{e}}+\beta_{\mathrm{e}}\left(v^{\prime}+\frac{1}{2}\right)$

$v_{v^{\prime \prime}, J^{\prime \prime}}^{v^{\prime}, J^{\prime}}=v_{v^{\prime}, v^{\prime \prime}}+F\left(J^{\prime}\right)-F\left(J^{\prime \prime}\right)$

where $B_{\mathrm{e}}, \alpha_{\mathrm{e}}, D_{\mathrm{e}}$, and $\beta_{\mathrm{e}}$ are spectroscopic constants. As discussed above, the rotational quantum numbers corresponding to the wavelengths $(387.6-388.6 \mathrm{~nm})$ range from 0 to 57 for the $(0,0)$ transition. In the case, the first term of Eq. (12) is $~ 90$ times the second term, and $B_{\mathrm{e}}$ is $\sim 100$ times $\alpha_{\mathrm{e}}$. Thus, the Eq. (12) can is derived as $F\left(J^{\prime}\right)=B_{\mathrm{e}} J^{\prime}\left(J^{\prime}+1\right)$

The first term of the transition frequency of each rotational line is much larger than the second term, and $v_{v^{\prime}, v^{\prime \prime}}$ is a constant for a given electronic transition, so the uncertainty due to $B_{\mathrm{e}}$ in $v_{v^{\prime \prime}, J^{\prime \prime}}^{v^{\prime}, J^{\prime}}$ is negligible, and the Eq. (11) can be described as

$\log \left(\frac{I_{J^{\prime \prime}}^{J^{\prime}}}{c_{3}}\right)=-\frac{B_{\mathrm{e}}}{T_{\mathrm{rot}}^{\prime}} \cdot c_{2}+c_{1}$

where $c_{1}, c_{2}$ and $c_{3}$ are three constants. As shown in the Eq. (17), we know $B_{\mathrm{e}}$ is the predominant spectroscopic constant affecting the estimated $T_{\text {rot }}$ and the systematic error in $T_{\text {rot }}$ is at the same order of magnitude as that of $B_{\mathrm{e}}$. Many researchers have estimated $B_{\mathrm{e}}$, and the results shows the difference in $B_{\mathrm{e}}$ is $<1 \%[22,23]$. Consequently, the systematic error in $T_{\text {rot }}$ estimated in this study is also $<1 \%$. More details and error analysis description of this method are given in the literature [17]. Then the vibrational temperature is estimated by minimizing the difference between the experimental and theoretical spectrum. In order to describe the measurement accuracy, we use the quantity $\mathrm{Er}$ to assess the degree of difference between the experimental and calculated spectrum [24].

In Fig. 6, the vibrational temperatures of the $\mathrm{CN}$ radical are obtained by fitting the profile of the experimental spectra of the $\mathrm{CN}$ violet system $\left(B^{2} \Sigma^{+} \rightarrow X^{2} \Sigma^{+}, \Delta v=0\right.$ sequence) to that of the theoretical spectra. The profiles in the solid line are the experimental spectra of the $\mathrm{CN}$ violet system at different delay times and the exposure time of the spectrometer acquiring system is $50 \mathrm{~ns}$. The profiles in the dotted line in Fig. 6 represent the corresponding theoretical synthetic spectra with the best-fit vibrational temperatures. The $E r$ on the top panel of Fig. 6a, b indicates that excellent agreements between the experimentally measured and the theoretical synthetic spectra are obtained and that reasonable rotational and vibrational temperature measurements are achieved. The results show that the shock-heated gas should reach thermal equilibrium at these two moments with the equilibrium temperature $T_{\mathrm{eq}}=7,400 \pm 300 \mathrm{~K}$. The accuracy of our experiments is similar with the results of other researchers by OES $[8,24]$. 

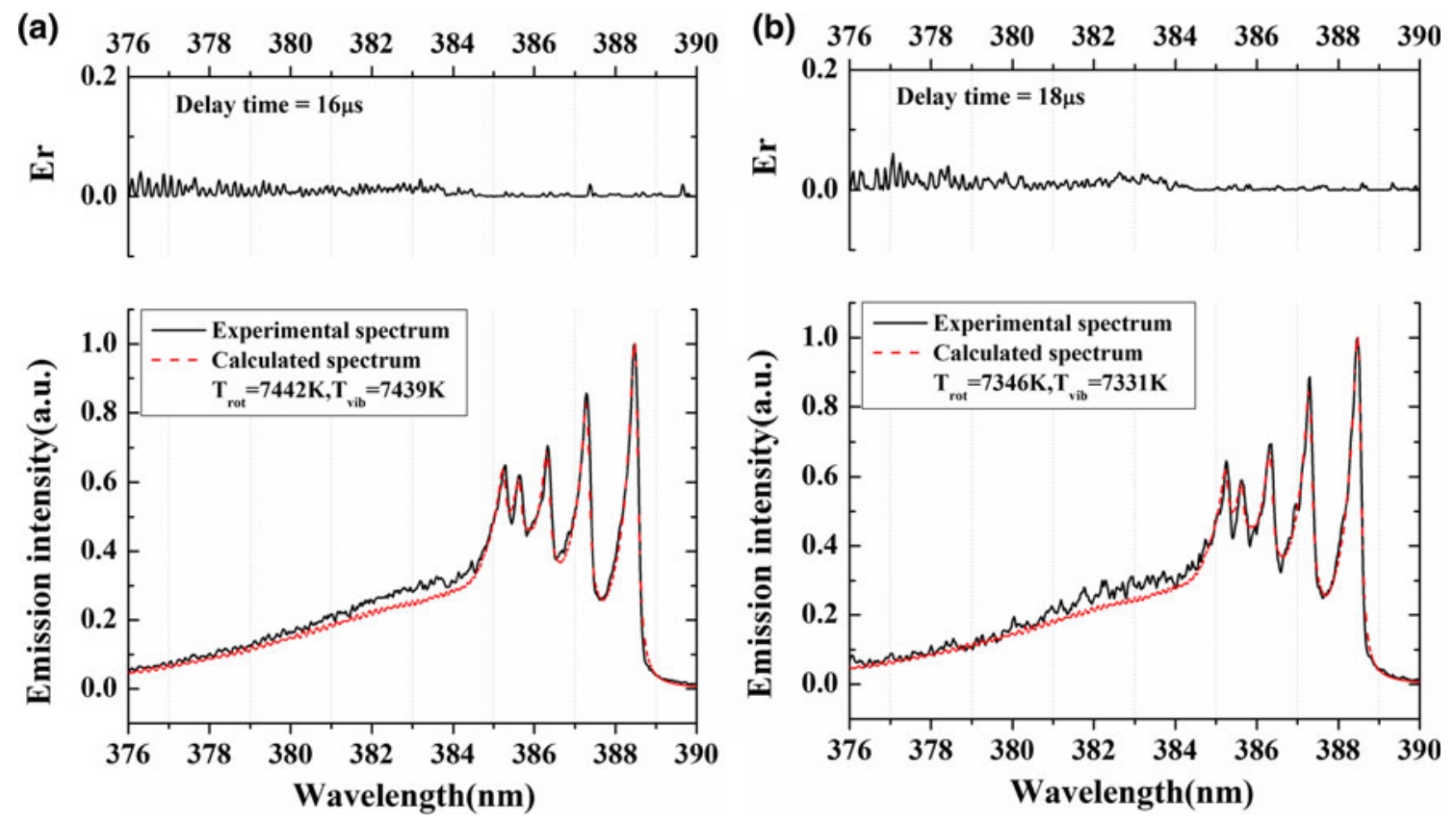

Fig. 6 Experimental and theoretical synthetic spectra of the $\mathrm{CN}$ violet system $\left(B^{2} \Sigma^{+} \rightarrow X^{2} \Sigma^{+}, \Delta v=0\right)$ at different delay times. (Experiment: $P_{1}=200 \mathrm{~Pa}, V_{\text {shock }}=6.31 \pm 0.11 \mathrm{~km} / \mathrm{s}$. Composition: $70 \% \mathrm{CO}_{2}$ and $30 \% \mathrm{~N}_{2}$.)

\section{3 $\mathrm{CO}$ absorption measurements behind the strong shock wave}

For shock tube experiments using TDLAS, measurement procedure is similar to the one described for the static cavity calibrations except that the TDLAS system is triggered by the No. 3 ion probe through a signal generator when the shock wave nears the probe. Figure 7 shows a representative absorption measurement signal behind a strong shock wave during one shot. In Fig. 7a, a sharp rising voltage output of the No. 3 ion probe indicates the arrival of the shock at the station of No. 3 probe location and propagates to the downstream region of the tube. According to the previous discussion, the shock-heated gas should reach thermal equilibrium after $16 \mu \mathrm{s}$ and the equilibrium temperature is equal to $7,400 \pm 300 \mathrm{~K}$, therefore the $\mathrm{CO}$ concentration can be calculated directly by means of the integrated absorbance, using Eq. (7). The data processing procedure usually follows this sequence: first, for each measured absorbance signal as shown in Fig. $7 \mathrm{~b}$, the non-absorbing wings are extracted and fitted into a polynomial, which is used as the baseline for constructing the absorbance plot of the absorption feature. The baseline polynomial order is adjusted until the baseline absorption signal is zero without any negative values. Here, the baseline is fitted with a fifth-order polynomial. Second, a Voigt profile is used to fit the absorbance plot in the first step. In addition, the chosen baseline also needs to yield a low residual between experiment and the Voigt fit data. The top panel of Fig. 8 shows a measured absorption line shape fitted with the Voigt profile, and the bottom portion shows the fitting residual, which is $<1 \%$ of the peak absorption throughout the line profile. Such low fittingresidual illustrates the high SNR is achieved in this research. Finally, based on the Voigt fit obtained in the second step, the integrated absorbance can be calculated, and then used to determine the $\mathrm{CO}$ concentration.

Figure 9 presents the evolutions of vibrational and rotational temperatures and integrated absorbance along the shock profile using OES and TDLAS, respectively. First, CN emission spectra have been obtained each $200 \mathrm{~ns}$ interval when the medium is considered out of equilibrium
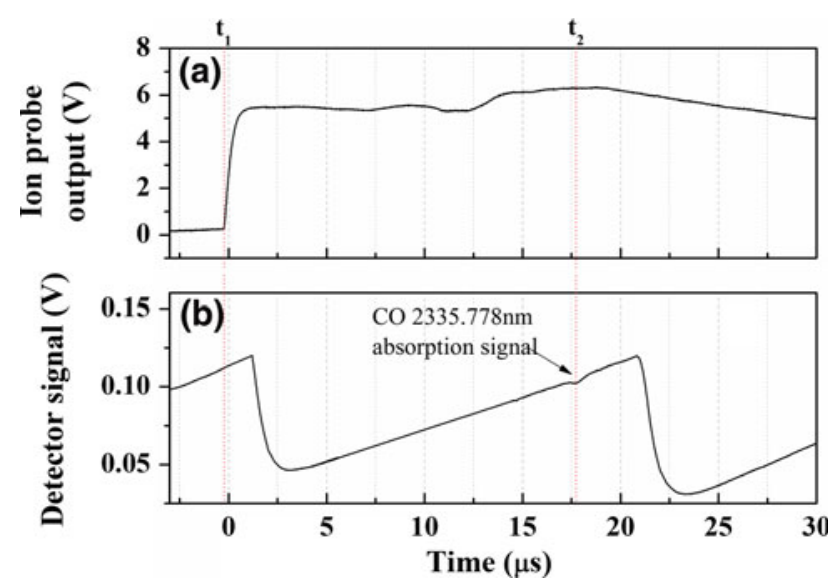

Fig. 7 a No. 3 ion probe output versus time and $\mathbf{b}$ corresponding absorption signal versus time under test conditions of $P_{1}=200 \mathrm{~Pa}$ and $V_{\text {shock }}=6.36 \mathrm{~km} / \mathrm{s}$ 

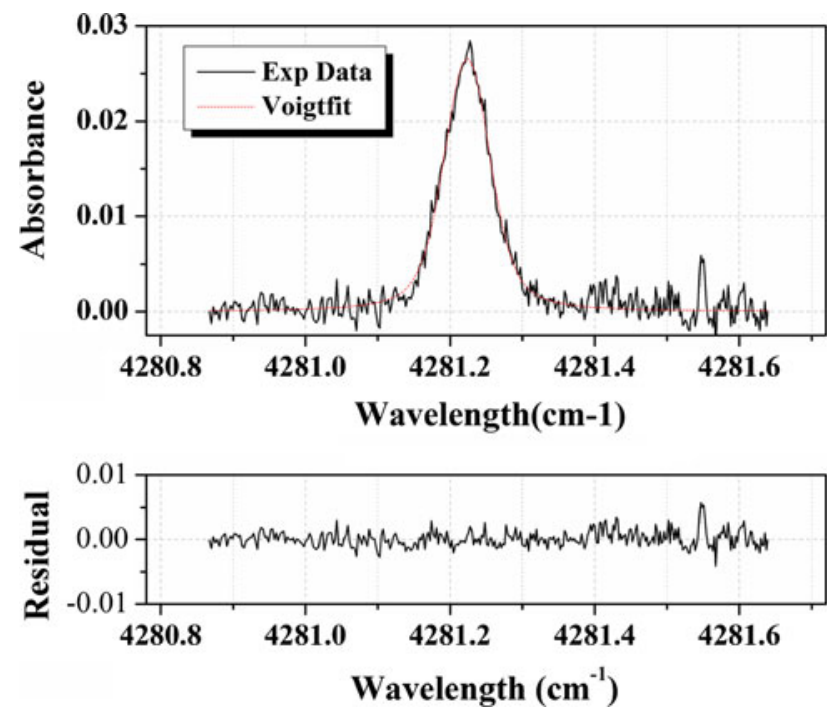

Fig. 8 Single-scan absorption data taken at $50 \mathrm{kHz}$ for concentration measurement in a shock tube with the $\mathrm{CO}$ transition near $2,335.778 \mathrm{~nm}$. Shown at the top is the best-fit Voigt profile to the experimental data, while the residual of the fit is shown at the bottom

and each $2 \mu$ s when equilibrium is assumed. The temperature decrease behind the shock front is clear and with a relatively large uncertainty on the temperature in the nonequilibrium region. Otherwise, the good agreement between rotational temperature and vibrational temperature after $16 \mu \mathrm{s}$ corresponds to an equilibrium temperature $T_{\text {eq }}=7,400 \pm 300 \mathrm{~K}$. Second, the slightly varied integrated absorbances of $\mathrm{CO}$ in the thermal equilibrium region means the $\mathrm{CO}$ concentration has also achieved equilibrium, then we can assume that both thermal and chemical equilibrium are achieved after $16 \mu$ s in our experimental conditions. The deduced average $\mathrm{CO}$ concentration is $7.46 \times 10^{12} \mathrm{~cm}^{-3}$, corresponding to the equilibrium temperature $7,400 \pm 300 \mathrm{~K}$. The integrated absorbance of $\mathrm{CO}$ in the non-equilibrium region can also determined by TDLAS, as shown in Fig. 9. However, non-Boltzmann distribution at ground state and components of the shockheated gas in the non-equilibrium region will lead some complexity for inferring $\mathrm{CO}$ concentration determination. Further study about temperature measurement using TDLAS for non-equilibrium state will be carried out in near future.

Uncertainty in the measurement concentration consists of the uncertainty in temperature measurement and uncertainty in the integrated absorbance. As shown in the Eq. (7), the uncertainty in temperature $(\sim 4 \%)$ can also affect the accuracy of line-strength calculation, a $4 \%$ error in the temperature creates a $\sim 5 \%$ the line-strength calculation error in our experimental temperature range. The uncertainty in the integrated absorbance is mainly caused by the error associated with baseline and Voigt profile fit.

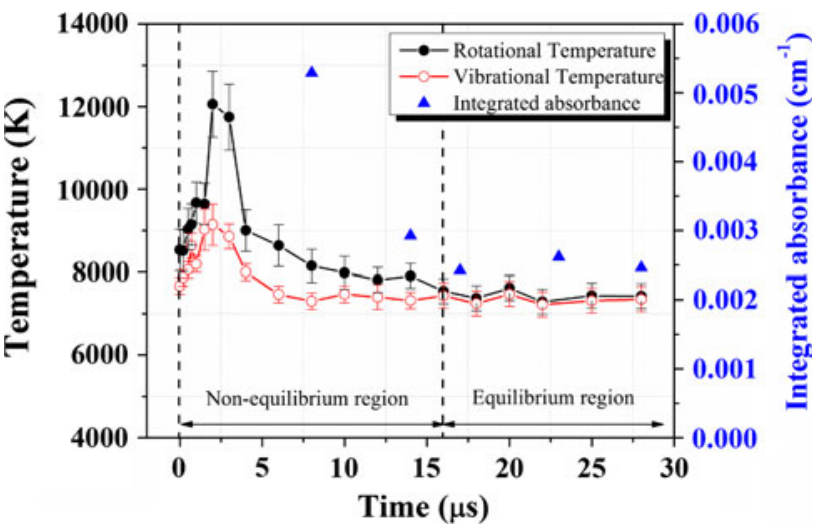

Fig. 9 Evolution of the $\mathrm{CO}$ integrated absorbance and temperatures behind the shock wave (Experiment: $P_{1}=200 \mathrm{~Pa}, V_{\text {shock }}=6.31 \pm$ $0.11 \mathrm{~km} / \mathrm{s}$. Composition: $70 \% \mathrm{CO}_{2}$ and $30 \% \mathrm{~N}_{2}$.)

Under our conditions, the high SNR of the baseline and Voigt profile fit, as indicated by the very small fitting residual shown in Fig. 8, results in an error of $\sim 1 \%$ in the integrated absorbance. Therefore, the uncertainty in the measure concentration is about $10 \%$. From the above discussion, we know temperature is the dominant parameter affecting the $\mathrm{CO}$ concentration measurement accuracy.

\section{Conclusions}

In this paper, experimental results concerning the $\mathrm{CO}$ concentration and gas temperature behind a strong shock wave in Martian-like mixture are presented. The experiments are conducted in a hydrogen-oxygen combustion driven shock tube using an optical diagnostic system based on OES and TDLAS. High temporal and spatial resolution experimental spectra of $\mathrm{CN}$ violet system $\left(B^{2} \Sigma^{+} \rightarrow X^{2} \Sigma^{+}\right.$, $\Delta v=0$ sequence) are used to determine the rotational and vibrational temperature distribution along the shock wave. Radiation structure of shock layer, including induction, relaxation and equilibrium processes can be also obtained through analysis of time gating optical emission spectra. The CO concentration is measured by a tunable diode laser absorption spectroscopy (TDLAS) system. For this TDLAS system, CO absorption line near $2.3 \mu \mathrm{m}$ is selected, and scanned-wavelength direct absorption mode is utilized with $50 \mathrm{kHz}$ scan rate. The average $\mathrm{CO}$ concentration in the thermal equilibrium region is deduced for the concerned test state. An error analysis has been performed to assess the uncertainties from the temperature measurement and the integrated absorbance. As we know, this is the first quantitative absorption measurement of $\mathrm{CO}$ concentration in relevant conditions for Mars atmospheric entry. The results will be very helpful to understand $\mathrm{CO}_{2}$ dissociation and provide verification data for computational fluid 
dynamic (CFD) simulation in high enthalpy Mars reentry chemical kinetics.

\section{References}

1. C. Park, J.T. Howe, R.L. Jaffe, G.V. Candler, Review of chemical kinetic problems of future NASA mission, II: Mars entries. J. Thermophys. Heat Transf. 8, 9-23 (1994)

2. S.P. Sharma, W. Gillespie, Nonequilibrium and equilibrium shock front radiation measurements. J. Thermophys. Heat Transf. 5, 257-265 (1991)

3. R.D. Braun, R.M. Manning, Mars exploration entry, descent, and landing challenges. J. Spacecraft Rockets 44, 310-323 (2007)

4. P. Boubert, C. Rond, Nonequilibrium radiation in shocked Martian mixtures. J. Thermophys. Heat Transf. 24, 40-49 (2010)

5. D. Bose, M.J. Wright, D.W. Bogdanoff, G.A. Raiche, G.A. Allen, Modeling and experimental assessment of $\mathrm{CN}$ radiation behind a strong shock wave. J. Thermophys. Heat Transf. 20, 220-230 (2006)

6. J.H. Grinstead, M.J. Wright, D.W. Bogdanoff, G.A. Allen, Shock radiation measurements for Mars aerocapture radiative heating analysis. J. Thermophys. Heat Transf. 23, 249-255 (2009)

7. C. Rond, P. Boubert, J.-M. Félio, A. Chikhaoui, Experimental investigation of radiative emission behind a strong shock wave in $\mathrm{N}_{2} / \mathrm{CH}_{4} / \mathrm{Ar}$ mixtures. AIAA 2006-3240, 37th AIAA Plasmadynamics and Lasers Conference (San Francisco, California, 2006)

8. C. Rond, P. Boubert, J.-M. Félio, A. Chikhaoui, Radiation measurements in a shock tube for Titan mixtures. J. Thermophys. Heat Transf. 21, 638-646 (2007)

9. C. Rond, P. Boubert, J.-M. Félio, A. Chikhaoui, Nonequilibrium radiation behind a strong shock wave in $\mathrm{CO}_{2}-\mathrm{N}_{2}$. Chem. Phys. 340, 93-104 (2007)

10. A.M. Brandis, R.G. Morgan, T.J. McIntyre, P.A. Jacobs, Nonequilibrium radiation intensity measurements in simulated Titan atmospheres. J. Thermophys. Heat Transf. 24, 291-300 (2010)

11. A.M. Brandis, R.G. Morgan, T.J. McIntyre, Analysis of nonequilibrium $\mathrm{CN}$ radiation encountered during Titan atmospheric entry. J. Thermophys. Heat Transf. 25, 493-499 (2011)
12. R.J. Stalker, Development of a hypervelocity wind tunnel. Aeronaut. J. 76, 374-384 (1972)

13. C. Rond, A. Bultel, P. Boubert, B.G. Chéron, Spectroscopic measurements of nonequilibrium $\mathrm{CO}_{2}$ plasma in $\mathrm{RF}$ torch. Chem. Phys. 354, 16-26 (2008)

14. Y. Babou, P. Rivière, M.-Y. Perrin, A. Soufiani, Spectroscopy study of microwave plasmas of $\mathrm{CO}_{2}$ and $\mathrm{CO}_{2}-\mathrm{N}_{2}$ mixtures at atmospheric pressure. Plasma Sources Sci. T. 17, 045010 (2008)

15. M.G. Allen, Diode laser absorption sensors for gas dynamic and combustion flows. Meas. Sci. Technol. 9, 545-562 (1998)

16. J.M. Meyers, D. Fletcher, Diode laser absorption sensor design and qualification for $\mathrm{CO}_{2}$ hypersonic flows. J. Thermophys. Heat Transf. 25, 193-200 (2011)

17. X. Lin, X.L. Yu, F. Li, S.H. Zhang, J.G. Xin, X.Y. Chang, Measurements of non-equilibrium and equilibrium temperature behind a strong shock wave in simulated Martian atmosphere. (be published in Acta Mech Sinica, doi: 10.1007/s10409-012-0104-9)

18. X. Chao, J.B. Jeffries, R.K. Hanson, Absorption sensor for CO in combustion gases using $2.3 \mu \mathrm{m}$ tunable diode lasers. Meas. Sci. Technol. 20, 115201 (2009)

19. A. Farooq, J.B. Jeffries, R.K. Hanson, CO2 concentration and temperature sensor for combustion gases using diode-laser absorption near $2.7 \mu \mathrm{m}$. Appl. Phys. B 90, 619-628 (2008)

20. R.R. Goldman, A. Gamache, J.-M. Perrin, C.P. Flaud, L.S. Rinsland, Rothman, HITRAN partition functions and weighted transition-moments squared. J. Quant. Spectrosc. Radiat. 66, 445-486 (2000)

21. D. Goorvitch, Infrared CO line list for the $X^{1} \Sigma^{+}$state. Astrophys. J. Suppl. S. 95, 535-552 (1994)

22. R.S. Ram, S.P. Davis, L. Wallace, R. Engleman, D.R.T. Appadoo, P.F. Bernath, Fourier transform emission spectroscopy of the $B^{2} \Sigma^{+}-X^{2} \Sigma^{+}$system of CN. J. Mol. Spectrosc. 237, 225-231 (2006)

23. C.V. Praced, P.F. Bernath, Fourier transform jet-emission spectroscopy of the $A^{2} \Pi-X^{2} \Sigma^{+}$transition of CN. J. Mol. Spectrosc. 156, 327-340 (1992)

24. K. Kurosawa, S. Sugita, K. Fujita, K. Ishibashi, T. Kadono, S. Ohno, T. Matsui, Rotational-temperature measurements of chemically reacting $\mathrm{CN}$ using band-tail spectra. J. Thermophys. Heat Transf. 23, 463-472 (2009) 